\title{
Correlating Schiff Moments in the Light Actinides with Octupole Moments
}

\author{
Jacek Dobaczewski, ${ }^{1,2,3,4}$ Jonathan Engel, ${ }^{5}$ Markus Kortelainen, ${ }^{2,4}$ and Pierre Becker ${ }^{1}$ \\ ${ }^{1}$ Department of Physics, University of York, Heslington, York YO10 5DD, United Kingdom \\ ${ }^{2}$ Department of Physics, University of Jyväskylä, P.O. Box 35 (YFL), FI-40014 Jyväskylä, Finland \\ ${ }^{3}$ Institute of Theoretical Physics, Faculty of Physics, University of Warsaw, ul. Pasteura 5, PL-02-093 Warsaw, Poland \\ ${ }^{4}$ Helsinki Institute of Physics, University of Helsinki, P.O. Box 64, FI-00014 Helsinki, Finland \\ ${ }^{5}$ Department of Physics and Astronomy, University of North Carolina, Chapel Hill, North Carolina 27516-3255, USA
}

(Received 24 July 2018; revised manuscript received 4 October 2018; published 4 December 2018)

\begin{abstract}
We show that the measured intrinsic octupole moments of ${ }^{220} \mathrm{Rn},{ }^{224} \mathrm{Ra}$, and ${ }^{226} \mathrm{Ra}$ constrain the intrinsic Schiff moments of ${ }^{225} \mathrm{Ra},{ }^{221} \mathrm{Rn},{ }^{223} \mathrm{Rn},{ }^{223} \mathrm{Fr},{ }^{225} \mathrm{Ra}$, and ${ }^{229} \mathrm{~Pa}$. The result is a dramatically reduced uncertainty in intrinsic Schiff moments. Direct measurements of octupole moments in odd nuclei will reduce the uncertainty even more. The only significant source of nuclear-physics error in the laboratory Schiff moments will then be the intrinsic matrix elements of the time-reversal noninvariant interaction produced by $C P$-violating fundamental physics. Those matrix elements are also correlated with octupole moments, but with a larger systematic uncertainty.
\end{abstract}

DOI: 10.1103/PhysRevLett.121.232501

The observation of a nonzero electric dipole moment (EDM) in a particle, atom, or molecule with a nondegenerate ground state would signal the violation of timereversal $(T)$ symmetry, which in any realistic field theory implies the violation of charge-parity $(C P)$ symmetry. The standard model violates both symmetries, of course, but at a level too low to be responsible for the lack of antimatter in the universe around us [1,2]. The asymmetry between matter and antimatter is apparently due to a stronger source of $C P$ violation, and the measurement of an atomic EDM is one of our best hopes to discover that source.

The atomic isotope with the best limit on its EDM is currently ${ }^{199} \mathrm{Hg}$. About 20 years ago, however, it was realized $[3,4]$ that atoms whose nuclei are asymmetrically shaped (octupole deformed, like pears) would have enhanced EDMs if the $C P$ violation occurred within the nucleus. The reason is connected to a partial screening of nuclear EDMs by electrons [5]. The argument goes as follows:

Because of the screening, the nuclear quantity that induces the atomic EDM is not the nuclear EDM itself, but rather the nuclear Schiff moment:

$S \equiv\left\langle\Psi_{0}\left|\hat{S}_{0}\right| \Psi_{0}\right\rangle \approx \sum_{i \neq 0} \frac{\left\langle\Psi_{0}\left|\hat{S}_{0}\right| \Psi_{i}\right\rangle\left\langle\Psi_{i}\left|\hat{V}_{P T}\right| \Psi_{0}\right\rangle}{E_{0}-E_{i}}+$ c.c.,

where $\left|\Psi_{0}\right\rangle$ is the member of the ground-state multiplet with the maximum angular-momentum $z$ projection, $\left|\Psi_{i}\right\rangle$

Published by the American Physical Society under the terms of the Creative Commons Attribution 4.0 International license. Further distribution of this work must maintain attribution to the author(s) and the published article's title, journal citation, and DOI. are excited states having the same angular-momentum quantum numbers as the ground state but opposite parity, and $\hat{S}_{0}$ is the Schiff operator

$$
\hat{S}_{0}=\frac{e}{10} \sqrt{\frac{4 \pi}{3}} \sum_{i}\left(r_{i}^{3}-\frac{5}{3} \overline{r_{\mathrm{ch}}^{2}} r_{i}\right) Y_{0}^{1}\left(\Omega_{i}\right)+\ldots
$$

Here the sum is over protons, $\overline{r_{\mathrm{ch}}^{2}}$ is the mean-square charge radius, and the omitted terms are smaller [6] and, to some extent, in dispute [6,7]. The operator $\hat{V}_{P T}$ in Eq. (1) is the $C P$-violating nucleon-nucleon interaction, to be discussed shortly. The asymmetric shape of octupole-deformed nuclei implies parity doubling (see, e.g., Ref. [8]): the presence of a partner $\left|\bar{\Psi}_{0}\right\rangle$ for the ground state $\left|\Psi_{0}\right\rangle$-with the same intrinsic structure and angular momentum but opposite parity - at a low excitation energy $\Delta E$. In ${ }^{225} \mathrm{Ra}$, e.g., the $1 / 2^{+}$ground state has a $1 / 2^{-}$partner at $55 \mathrm{keV}$ [9]. The similarity of the two partner states and the low excitation energy means not only that the partner dominates the sum in Eq. (1), leading to the quite accurate approximation,

$$
S \approx-2 \frac{\left\langle\Psi_{0}\left|\hat{S}_{0}\right| \bar{\Psi}_{0}\right\rangle\left\langle\bar{\Psi}_{0}\left|\hat{V}_{P T}\right| \Psi_{0}\right\rangle}{\Delta E},
$$

but also that it enhances the Schiff moment by large amounts over the moments in nuclei with symmetric shapes.

Much of the enhancement is due to the small energy denominator $\Delta E$, but some comes from the presence in the numerator of the Schiff operator rather than the electric dipole operator. Dipole moments are delicate because they depend on the difference between the center of mass and center of charge, which is often small even in octupoledeformed nuclei. Because of the radial weighting in Eq. (2), 
however, Schiff moments can be substantial even if the centers of mass and charge coincide.

The expectation value of the first term in Eq. (2) is much larger in octupole-deformed nuclei than that of the second term, which is proportional to the EDM. In fact, if the spherical harmonic $Y_{0}^{1}$ were replaced by $Y_{0}^{3}$, that first term would just be proportional to the octupole charge operator [10]: $\hat{Q}_{0}^{3} \equiv e \sum_{i} r_{i}^{3} Y_{0}^{3}(i)$, where the sum again is over protons. The matrix elements of this operator are a direct measure of octupole deformation and thus not at all delicate in octupole-shaped isotopes.

This argument has an obvious implication: the Schiff moment should be correlated with the matrix elements of the octupole moment, and measured octupole transition rates should allow us to reduce the uncertainty in calculations of Schiff moments. Such calculations are essential if we want to use limits on atomic EDMs (or an eventual observation of one) to make quantitative statements about new sources of $C P$ violation. Existing calculations [16] carry an uncertainty [17] that is significantly larger than $100 \%$, and the use of complementary measurements to exploit them is important. In the rest of this Letter we show that measured octupole properties are a great help.

The story is not quite as simple as it first appears, however, partly because the Schiff operator is not the only ingredient in Eq. (1); the $C P$-violating potential $V_{\mathrm{PT}}$, which atomic EDM experiments hope to elucidate, also plays a role. The potential, which is often discussed in terms of meson exchange, can be represented in chiral effective field theory [18], a QCD-based picture of interacting nucleons and pions that has a systematic powercounting scheme. Including the most important terms, one has [18-20],

$$
\begin{aligned}
\hat{V}_{\mathrm{PT}}\left(\boldsymbol{r}_{1}-\boldsymbol{r}_{2}\right)= & -\frac{g m_{\pi}^{2}}{8 \pi m_{N}}\left\{\left(\boldsymbol{\sigma}_{1}-\boldsymbol{\sigma}_{2}\right) \cdot\left(\boldsymbol{r}_{1}-\boldsymbol{r}_{2}\right)\left[\bar{g}_{0} \vec{\tau}_{1} \cdot \vec{\tau}_{2}-\frac{\bar{g}_{1}}{2}\left(\tau_{1 z}+\tau_{2 z}\right)+\bar{g}_{2}\left(3 \tau_{1 z} \tau_{2 z}-\vec{\tau}_{1} \cdot \vec{\tau}_{2}\right)\right]\right. \\
& \left.-\frac{\bar{g}_{1}}{2}\left(\boldsymbol{\sigma}_{1}+\boldsymbol{\sigma}_{2}\right) \cdot\left(\boldsymbol{r}_{1}-\boldsymbol{r}_{2}\right)\left(\tau_{1 z}-\tau_{2 z}\right)\right\} \frac{\exp \left(-m_{\pi}\left|\boldsymbol{r}_{1}-\boldsymbol{r}_{2}\right|\right)}{m_{\pi}\left|\boldsymbol{r}_{1}-\boldsymbol{r}_{2}\right|^{2}}\left[1+\frac{1}{m_{\pi}\left|\boldsymbol{r}_{1}-\boldsymbol{r}_{2}\right|}\right] \\
& +\frac{1}{2 m_{N}^{3}}\left[\bar{c}_{1}+\bar{c}_{2} \vec{\tau}_{1} \cdot \vec{\tau}_{2}\right]\left(\boldsymbol{\sigma}_{1}-\boldsymbol{\sigma}_{2}\right) \cdot \boldsymbol{\nabla} \delta^{3}\left(\boldsymbol{r}_{1}-\boldsymbol{r}_{2}\right),
\end{aligned}
$$

where arrows denote isovector operators, $\tau_{z}$ is +1 for neutrons, $m_{N}$ is the nucleon mass, and (in this equation only) we use the convention $\hbar=c=1$. The $\bar{g}$ 's are the unknown isoscalar, isovector, and isotensor $T$-violating pion-nucleon coupling constants, the $\bar{c}$ 's are the unknown coupling constants of a short-range interaction that subsumes the effects of heavy-meson exchange, and $g$ is the usual strong $\pi N N$ coupling constant. Most calculations thus far have neglected the effects of the contact interactions in the last line of Eq. (4) (as well as the effects of neutron and proton EDMs). Here we write the Schiff moment $S$ in Eq. (1) as

$$
S=a_{0} g \bar{g}_{0}+a_{1} g \bar{g}_{1}+a_{2} g \bar{g}_{2}+b_{1} \bar{c}_{1}+b_{2} \bar{c}_{2} .
$$

The coefficients $a_{i}$ and $b_{i}$, which are the result of a calculation, have units $e \mathrm{fm}^{3}$.

Another slight complication comes from our use of Skyrme [21,22] or Gogny [22,23] energy-density functional theory (related to mean-field theory) to express the nuclear wave function in terms of a deformed and paritymixed Slater determinant or a more general deformed quasiparticle vacuum. The deformed wave function represents the intrinsic state of the nucleus $\left|\Phi_{0}\right\rangle$, that is, the nuclear state in a body-fixed frame. The Schiff moment in this frame is independent of $\hat{V}_{\text {PT }}$, the function of which, in a manner of speaking, is to ensure that the intrinsic breaking of parity and time-reversal symmetries by mean-field theory survives in the laboratory frame. It is the intrinsic Schiff moment that we can most easily correlate with measured octupole transition rates, as we now explain:

Having obtained an intrinsic state through mean-fieldlike calculations, one needs to project it onto laboratory states with well-defined angular momentum and parity, in our case the two states in the parity doublet that determine the laboratory Schiff moment. In our prior work on the subject, described in Ref. [16], and in the measurements of deformation, shapes are assumed to be infinitely rigid. This approximation leads to the well-known result [24] that all of the ground-band reduced matrix elements of an (arbitrary) operator $\hat{X}^{\lambda}$ with multipolarity $\lambda$ are proportional to an intrinsic-state expectation value $\left\langle\hat{X}_{0}^{\lambda}\right\rangle$ :

$$
\left\langle J|| \hat{X}^{\lambda}|| J^{\prime}\right\rangle_{\text {rigid }}=\sqrt{2 J^{\prime}+1}\left\langle J^{\prime} K, \lambda 0 \mid J K\right\rangle\left\langle\hat{X}_{0}^{\lambda}\right\rangle,
$$

where $\left\langle J^{\prime} K, \lambda 0 \mid J K\right\rangle$ is a Clebsch-Gordan coefficient and the intrinsic-state expectation value $\left\langle X_{0}^{\lambda}\right\rangle$ is evaluated in an axially symmetric state having angular-momentum projection $K$ on the symmetry axis. The Wigner-Eckart theorem then implies that the observable laboratory transition matrix elements 


$$
\left\langle J M\left|\hat{X}_{\mu}^{\lambda}\right| J^{\prime} M^{\prime}\right\rangle=\left\langle J^{\prime} M^{\prime}, \lambda \mu \mid J M\right\rangle \frac{\left\langle J|| \hat{X}^{\lambda}|| J^{\prime}\right\rangle}{\sqrt{2 J+1}},
$$

can be used to extract values of $\left\langle\hat{X}_{0}^{\lambda}\right\rangle$ simply in the rigiddeformation limit. With the Clebsch-Gordan coefficients evaluated, Eqs. (6) and (7) relate $\left\langle\Psi_{0}\left|\hat{S}_{0}\right| \bar{\Psi}_{0}\right\rangle$ to the intrinsic expectation value $S_{0} \equiv\left\langle\hat{S}_{0}\right\rangle$ and $\left\langle\Psi_{0}\left|\hat{V}_{\mathrm{PT}}\right| \bar{\Psi}_{0}\right\rangle$ to the intrinsic expectation value $\left\langle\hat{V}_{\mathrm{PT}}\right\rangle$ :

$$
\begin{aligned}
\left\langle\Psi_{0}\left|\hat{S}_{0}\right| \bar{\Psi}_{0}\right\rangle_{\text {rigid }} & =\frac{J}{J+1} S_{0}, \\
\left\langle\bar{\Psi}_{0}\left|\hat{V}_{\mathrm{PT}}\right| \Psi_{0}\right\rangle_{\text {rigid }} & =\left\langle\hat{V}_{\mathrm{PT}}\right\rangle,
\end{aligned}
$$

where Eq. (8) is specific to the case $J=J^{\prime}=M=M^{\prime}=K$. Equations (6) and (7) also relate octupole transition rates to the intrinsic octupole moment $Q_{0}^{3} \equiv\left\langle\hat{Q}_{0}^{3}\right\rangle$.

In fact, we are no longer confined to this rigid-deformation limit; we can now obtain the ground state and its partner by exactly projecting the lowest intrinsic state onto states with any angular momentum and with positive or negative parity. Thus, we can test the quality of the rigid-deformation approximation by comparing reduced matrix elements from Eq. (6) to those evaluated exactly between angularmomentum and parity-projected states. For the Schiff operator the rigid-deformation approximation turns out to be very good; it induces an error of only about $1.5 \%$. The rigid-deformation approximation for the octupole operator $\hat{Q}^{3}$ in ${ }^{224} \mathrm{Ra}$ is even better, inducing an error of less than $0.1 \%$.

The error from extrapolating our results to a singleparticle space with an infinite number of harmonic-oscillator shells is also quite small, about $0.02 \%$. For calculations with 20 oscillator shells, which lead to a reasonable balance between CPU time and precision, the two errors have similar magnitude and opposite sign. We will thus use this basis for all calculations performed with Skyrme functionals. The Gogny functional leads to CPU time that is much longer; we therefore settle for 16 oscillator shells when working with it. We use the code HFODD (v2.84h) $[25,26]$ to carry out all Hartree-Fock (HF), Hartree-FockBogolyubov (HFB), or BCS calculations. The accuracy of all these approximations means that we can consider the Schiff and octupole transition matrix elements to be directly proportional to the corresponding intrinsic moments, the correlation of which we now address in more detail.

Figure 1(a) shows the unconstrained predictions of many functionals, with and without pairing (that is, in the HF or HFB/BCS approximations), for the octupole and Schiff moments of ${ }^{225} \mathrm{Ra}$. The correlation between the two observables is striking, good enough so that one can nearly identify a unique prediction for the intrinsic Schiff moment, given a measured octupole moment. We say "nearly" because neutron pairing, the exact strength of which is unknown, introduces some ambiguity. The red dots correspond to a neutron pairing gap of $0.747 \mathrm{MeV}$, a sensible

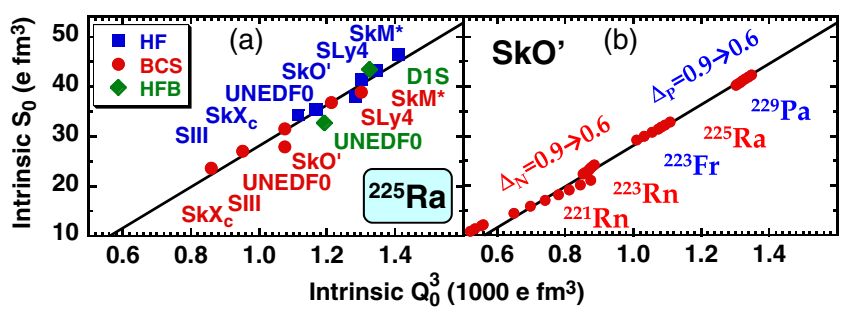

FIG. 1. (a) The unconstrained predictions of the functionals $\mathrm{SkX}_{c}$ [27], SIII [28], UNEDF0 [29], SkO' [30], SLy4 [31], SkM* [32], and D1S [33] for the octupole and Schiff moments of ${ }^{225} \mathrm{Ra}$, with and without pairing. (b) Predictions of $\mathrm{SkO}^{\prime}$ for the octupole and Schiff moments in ${ }^{221} \mathrm{Rn},{ }^{223} \mathrm{Rn},{ }^{223} \mathrm{Fr},{ }^{225} \mathrm{Ra}$, and ${ }^{229} \mathrm{~Pa}$, with varying amounts of neutron $(N)$ or proton $(P)$ pairing. In odd- $N$ or odd- $Z$ nuclei, the moments increase as the pairing gaps decrease from $\Delta_{N(P)}=0.90$ to $0.70 \mathrm{MeV}$, in steps of $0.05 \mathrm{MeV}$.

value determined by the odd-even mass staggering around ${ }^{225} \mathrm{Ra}$. Figure 1(b) shows the range of predictions with the functional $\mathrm{SkO}^{\prime}$ for the reasonable range $0.6 \mathrm{MeV} \leq$ $\Delta_{N(P)} \leq 0.9 \mathrm{MeV}$ in five odd- $A$ nuclei with large asymmetric deformation. The correlation between Schiff and octupole moments is quite linear and it allows the identification of an uncertainty for the predictions $\mathrm{SkO}^{\prime}$.

All these results indicate the desirability of measuring $Q_{0}^{3}$ in odd nuclei for which atomic EDM measurements are conceivable. Though no one has made such a measurement (yet), the Liverpool group reported the measurement of $Q_{0}^{3}$ in the neighboring even-even nucleus ${ }^{224} \mathrm{Ra}$ a few years ago [15]. Figure 2 shows the same kind of results as does Fig. 1, but with the predicted intrinsic Schiff moments plotted versus the octupole moment of ${ }^{224} \mathrm{Ra}$ and with several Skyrme functionals instead of several nuclei in panel (b). The correlation of the two moments is still evident in both panels. The vertical band in the two panels represents the

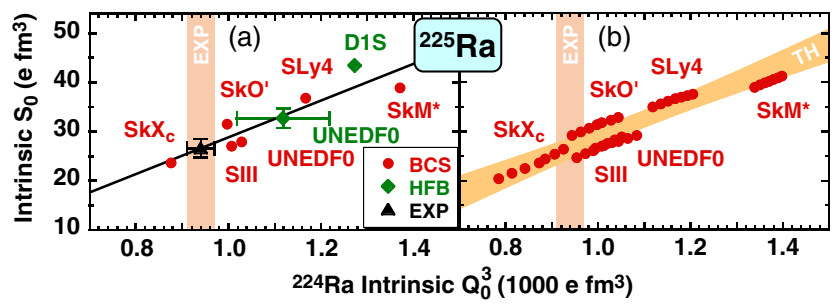

FIG. 2. Panel (a) is like the corresponding panel of Fig. 1 (with the same value for the neutron pairing gap) but with the octupole moment of ${ }^{224} \mathrm{Ra}$ on the abscissa and its experimental error represented by the vertical bar. The results for UNEDF0, for which a full covariance matrix was determined [29] include a statistical uncertainty. Panel (b), like the same panel in Fig. 1, plots the variation of the Schiff and octupole moments when neutron and proton pairing gaps are varied between 0.9 and $0.6 \mathrm{MeV}$, but in a single nucleus and for a variety of Skyrme functionals. The theoretical error band (TH) represents the result of a regression analysis [34]. 


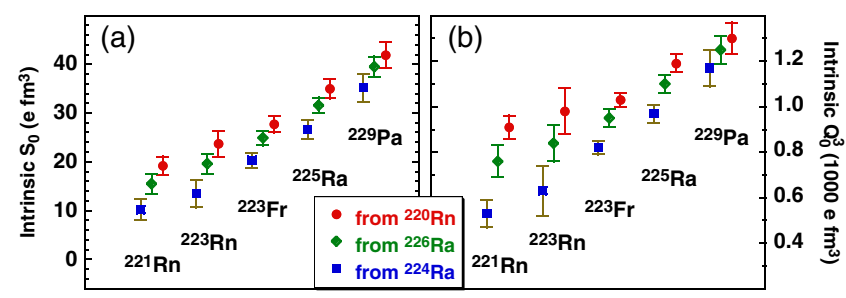

FIG. 3. Intrinsic Schiff moments $S_{0}$ in e $\mathrm{fm}^{3}$ (a) and octupole moments $Q_{0}^{3}$ in units of $1000 e \mathrm{fm}^{3}$ (b) of ${ }^{221} \mathrm{Rn},{ }^{223} \mathrm{Rn},{ }^{223} \mathrm{Fr}$, ${ }^{225} \mathrm{Ra}$, and ${ }^{229} \mathrm{~Pa}$, determined from the experimental octupole moments of ${ }^{224} \mathrm{Ra},{ }^{226} \mathrm{Ra}$, and ${ }^{220} \mathrm{Rn}$.

measured value of the ${ }^{224} \mathrm{Ra}$ octupole moment, with its width and that of the error bar on top of it representing experimental uncertainty. The slanted line in Fig. 2(a) and band in Fig. 2(b) represent, respectively, the correlation and total uncertainty [34] of the Schiff moment. The uncertainty is smaller near the middle of the figure, where the calculated moments have more impact.

Figure 2(a) contains, in addition to the items already discussed, the results of a quantitative analysis in black. We use linear regression to determine the coefficients $a$ and $b$ in the relation $S_{10}=a+b \times Q_{0}^{3}\left({ }^{224} \mathrm{Ra}\right)$. The Supplemental Material [34] for this manuscript provides more details. For ${ }^{225} \mathrm{Ra}$, the propagated intrinsic Schiff moment and its uncertainty at the experimental intrinsic octupole moment $Q_{0}^{3}\left({ }^{224} \mathrm{Ra}\right)=940(30)$ is $S_{0}=26.6(1.9) \mathrm{efm}^{3}$. The theoretical uncertainty of $1.6 \mathrm{e} \mathrm{fm}^{3}$ is larger than that from experiment, which is $1.1 \mathrm{e} \mathrm{fm}^{3}$.

It is now clear that the observed correlation between the calculated intrinsic Schiff moment in ${ }^{225} \mathrm{Ra}$ and octupole moment in ${ }^{224} \mathrm{Ra}$ allows us to greatly reduce systematic uncertainties stemming from nuclear functionals. Figure 3(a) shows predictions for the intrinsic Schiff moments of ${ }^{221} \mathrm{Rn}$, ${ }^{223} \mathrm{Rn},{ }^{223} \mathrm{Fr},{ }^{225} \mathrm{Ra}$, and ${ }^{229} \mathrm{~Pa}$ from the experimental octupole moments of ${ }^{224} \mathrm{Ra}$ [15], ${ }^{226} \mathrm{Ra}$ [38], and ${ }^{220} \mathrm{Rn}$ [15]. A similar analysis, shown in Fig. 3(b), allows us to predict values of octupole moments in these odd nuclei. Numerical values for all these intrinsic moments are collected in the Supplemental Material [34]. The discrepancies that remain among predictions reflect systematic uncertainty that our analysis has not removed.

To obtain an independent estimate of systematic uncertainties in the intrinsic Schiff moment of ${ }^{225} \mathrm{Ra}$, we employ the full covariance matrix of the UNEDF0 functional model parameters [29]. This gives an intrinsic Schiff moment in ${ }^{225} \mathrm{Ra}$ of $S_{10}=32.7(1.9) \mathrm{e} \mathrm{fm}^{3}$ and an octupole moment in ${ }^{224} \mathrm{Ra}$ of $Q_{0}^{3}=1.17(10) \times 1000 \mathrm{e} \mathrm{fm}^{3}$ [cf. the error bars in Fig. 2(b)]. It also yields a very strong correlation coefficient of 0.908 between the two moments. The relatively large uncertainty in $Q_{0}^{3}$ means that only a modest increase in the UNEDF0 penalty function is required to alter the coupling constants so that the calculated $Q_{0}^{3}$ agrees with experiment.

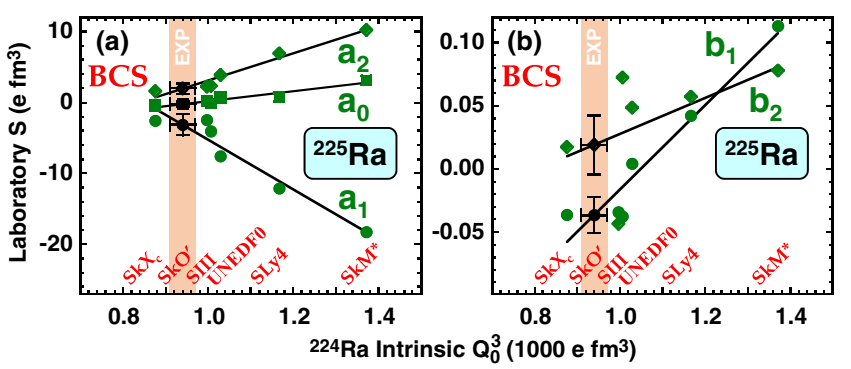

FIG. 4. Coefficients $a_{0}, a_{1}$, and $a_{2}$ (a) and $b_{1}$ and $b_{2}$ (b), Eq. (5), corresponding to the finite and zero-range terms of $\hat{V}_{\text {PT }}$, Eq. (4), determined in ${ }^{225} \mathrm{Ra}$ for six Skyrme functionals and propagated to the experimental value of the octupole moment in ${ }^{224} \mathrm{Ra}$.

The strong correlation between the octupole and Schiff moments then means that the Schiff moment would probably slide closer to our propagated value of $26.6 \mathrm{em}^{3}$. This hypothetical result, however, can only be verified by a full refit of UNEDF0 with the experimental value of $Q_{0}^{3}$ in ${ }^{224} \mathrm{Ra}$ included in the penalty function.

So far all our focus has been on the intrinsic Schiff moment, which, as we have noted, is only one of the two ingredients in the laboratory Schiff moment (3); the other is the intrinsic matrix element of $\hat{V}_{\text {PT }}$. Can we use measured octupole moments to constrain it as well? Figure 4 shows the variation of coefficients $a_{0}, a_{1}, a_{2}, b_{1}$, and $b_{2}$, Eq. (5), (In Refs. [16,39] the signs of coefficients $a_{0}, a_{1}$, and $a_{2}$ were inverted.) in ${ }^{225} \mathrm{Ra}$ with the octupole moment in ${ }^{224} \mathrm{Ra}$. Apart from $b_{2}$, there is a clear correlation that allows for a meaningful extrapolation to the measured value; a larger scatter of points induces a larger extrapolation error for $b_{2}$.

The analysis becomes more complicated, however, when we include measured octupole moments from other isotopes. The correlation between a given octupole moment and the coefficients $a_{i}, b_{i}$ still exists, but the use of two different octupole moments to constrain the coefficients can lead to quite different values. This situation is unlike that depicted by Fig. 3, and suggests the presence of significant systematic error in the calculations of the intrinsic matrix element of $\hat{V}_{\text {PT }}$.

The figures in our Supplemental Material [34] show that the correlation between an octupole moment in one nucleus and a laboratory Schiff moment in another is better if the two nuclei are very close together in $Z$ and $N$. We therefore use only the octupole moment of ${ }^{220} \mathrm{Ra}$ in computing the coefficients $a_{i}$ and $b_{i}$ in ${ }^{221} \mathrm{Rn}$ and ${ }^{223} \mathrm{Rn}$, and only the moments of ${ }^{224} \mathrm{Ra}$ and ${ }^{226} \mathrm{Ra}$ when computing the coefficients in ${ }^{223} \mathrm{Fr},{ }^{225} \mathrm{Ra}$, and ${ }^{229} \mathrm{~Pa}$. As Table I shows, we still end up with a sizable uncertainty in the coefficients, even with these restrictions. The numbers in italics there are consistent with zero, and only those in boldface are determined with a precision of $25 \%$ or better. In ${ }^{225} \mathrm{Ra}$, our central values for $a_{1}$ and $a_{2}$ are slightly smaller than in our earlier computation [16], while $a_{0}$ is consistent with 
TABLE I. Coefficients $a_{0}, a_{1}, a_{2}, b_{1}$, and $b_{2}$ (in e $\mathrm{fm}^{3}$ ) [from Eq. (5)], determined by regression analysis. For ${ }^{221} \mathrm{Rn}$ and ${ }^{223} \mathrm{Rn}$ we show values propagated to the experimental octupole moment of ${ }^{220} \mathrm{Rn}$, whereas for ${ }^{223} \mathrm{Fr},{ }^{225} \mathrm{Ra}$, and ${ }^{229} \mathrm{~Pa}$ we show averages of those propagated to ${ }^{224} \mathrm{Ra}$ and ${ }^{226} \mathrm{Ra}$. Details are in the Supplemental Material [34]. Values determined with a precision better than $25 \%$ are in boldface and those compatible with zero are in italics.

\begin{tabular}{cccccc}
\hline \hline & $a_{0}$ & $a_{1}$ & $a_{2}$ & $b_{1}$ & $b_{2}$ \\
\hline${ }^{221} \mathrm{Rn}$ & $-0.04(10)$ & $\mathbf{- 1 . 7 ( 3 )}$ & $\mathbf{0 . 6 7}(\mathbf{1 0})$ & $-0.015(5)$ & $-0.007(4)$ \\
${ }^{223} \mathrm{Rn}$ & $-0.08(8)$ & $\mathbf{- 2 . 4 ( 4 )}$ & $\mathbf{0 . 8 6}(\mathbf{1 0})$ & $-0.031(9)$ & $-0.008(8)$ \\
${ }^{223} \mathrm{Fr}$ & $0.07(20)$ & $-0.8(7)$ & $0.05(40)$ & $0.018(8)$ & $-0.016(10)$ \\
${ }^{225} \mathrm{Ra}$ & $0.2(6)$ & $-5(3)$ & $3.3(1.5)$ & $-0.01(3)$ & $0.03(2)$ \\
${ }^{229} \mathrm{~Pa}$ & $\mathbf{- 1 . 2 ( 3 )}$ & $-0.9(9)$ & $-0.3(5)$ & $\mathbf{0 . 0 3 6}(\mathbf{8})$ & $0.032(18)$ \\
\hline \hline
\end{tabular}

zero. Note that only in this nucleus and in ${ }^{223} \mathrm{Fr}$ were we able to use the experimental excitation energy in Eq. (3); in the other isotopes, because reliable measurements are not available, we took the excitation energy to be $100 \mathrm{keV}$. As Eq. (3) shows, when data become available, the results in the table can be simply scaled.

How does one reduce the uncertainty in the laboratory Schiff moments? The obvious way is to isolate and measure a quantity that is closely correlated with the intrinsic matrix element of $\hat{V}_{\text {PT }}$. Although that potential is a two-body operator, it can be approximated by an average one-body operator with the schematic form $\boldsymbol{\sigma} \cdot \boldsymbol{r}$, as, e.g., in Refs. [3,39]. Such operators (and related two-body meson-exchange versions) occur within subleading pieces of the hadronic electroweak current, but identifying and measuring the appropriate matrix elements will be a challenge. The potential payoff, however, makes it worth addressing.

We thank Michael Ramsey-Musolf and Peter Butler for useful discussions. This work was supported by the U.S. Department of Energy through Contract No. DE-FG0297ER41019, by the STFC Grants No. ST/M006433/1 and No. ST/P003885/1, and by the Academy of Finland and University of Jyväskylä within the FIDIPRO program. We acknowledge the CSC-IT Center for Science Ltd., Finland, for the allocation of computational resources.

[1] D. E. Morrissey and M. J. Ramsey-Musolf, New J. Phys. 14, 125003 (2012).

[2] M. Dine and A. Kusenko, Rev. Mod. Phys. 76, 1 (2003).

[3] N. Auerbach, V. V. Flambaum, and V. Spevak, Phys. Rev. Lett. 76, 4316 (1996).

[4] J. Engel, J. L. Friar, and A. C. Hayes, Phys. Rev. C 61, 035502 (2000).

[5] L. I. Schiff, Phys. Rev. 132, 2194 (1963).

[6] R. A. Sen'kov, N. Auerbach, V. V. Flambaum, and V. G. Zelevinsky, Phys. Rev. A 77, 014101 (2008).
[7] C.-P. Liu, M. J. Ramsey-Musolf, W. C. Haxton, R. G. E. Timmermans, and A.E. L. Dieperink, Phys. Rev. C 76, 035503 (2007).

[8] R. Sheline, A. Jain, K. Jain, and I. Ragnarsson, Phys. Lett. B 219, 47 (1989).

[9] R. Helmer, M. Lee, C. Reich, and I. Ahmad, Nucl. Phys. A474, 77 (1987).

[10] In this Letter, we use the definition of Ref. [11]. The definitions $\hat{Q}_{0}^{3}=e \sqrt{4 \pi / 7} \sum_{i} r_{i}^{3} Y_{0}^{3}(i) \quad$ [12] and $\hat{Q}_{0}^{3}=$ $e \sqrt{16 \pi / 7} \sum_{i} r_{i}^{3} Y_{0}^{3}(i)[13,14]$ also appear in the literature. The experimental value of the ${ }^{224} \mathrm{Ra}$ spectroscopic octupole moment measured in Ref. [15], $2520 \pm 90 \mathrm{e} \mathrm{fm}^{3}$ requires the definition of Refs. [13,14]. With our definition, this number becomes $Q_{0}^{3}=940 \pm 30 \mathrm{e} \mathrm{fm}^{3}$.

[11] A. Bohr and B. R. Mottelson, Nuclear Structure (W.A. Benjamin, New York, 1969), Vol. I.

[12] L. M. Robledo and G. F. Bertsch, Phys. Rev. C 86, 054306 (2012)

[13] G. A. Leander and Y. S. Chen, Phys. Rev. C 37, 2744 (1988).

[14] P. A. Butler and W. Nazarewicz, Rev. Mod. Phys. 68, 349 (1996).

[15] L. P. Gaffney et al., Nature (London) 497, 199 (2013).

[16] J. Dobaczewski and J. Engel, Phys. Rev. Lett. 94, 232502 (2005).

[17] J. Engel, M. J. Ramsey-Musolf, and U. van Kolck, Prog. Part. Nucl. Phys. 71, 21 (2013).

[18] C. M. Maekawa, E. Mereghetti, J. de Vries, and U. van Kolck, Nucl. Phys. A872, 117 (2011).

[19] W. C. Haxton and E. M. Henley, Phys. Rev. Lett. 51, 1937 (1983).

[20] P. Herczeg, Hyperfine Interact. 43, 75 (1988).

[21] T. H. R. Skyrme, Nucl. Phys. 9, 615 (1958).

[22] M. Bender, P.-H. Heenen, and P.-G. Reinhard, Rev. Mod. Phys. 75, 121 (2003).

[23] J. Dechargé and D. Gogny, Phys. Rev. C 21, 1568 (1980).

[24] A. Bohr and B. R. Mottelson, Nuclear Structure (W.A. Benjamin, Reading, 1975), Vol. II.

[25] N. Schunck, J. Dobaczewski, W. Satuła, P. Bączyk, J. Dudek, Y. Gao, M. Konieczka, K. Sato, Y. Shi, X. Wang, and T. Werner, Comput. Phys. Commun. 216, 145 (2017).

[26] J. Dobaczewski et al. (to be published).

[27] B. A. Brown, Phys. Rev. C 58, 220 (1998).

[28] M. Beiner, H. Flocard, N. V. Giai, and P. Quentin, Nucl. Phys. A238, 29 (1975).

[29] M. Kortelainen, T. Lesinski, J. Moré, W. Nazarewicz, J. Sarich, N. Schunck, M. V. Stoitsov, and S. Wild, Phys. Rev. C 82, 024313 (2010).

[30] P.-G. Reinhard, Nucl. Phys. A649, 305 (1999).

[31] E. Chabanat, P. Bonche, P. Haensel, J. Meyer, and R. Schaeffer, Nucl. Phys. A635, 231 (1998).

[32] J. Bartel, P. Quentin, M. Brack, C. Guet, and H.-B. Håkansson, Nucl. Phys. A386, 79 (1982).

[33] J. Berger, M. Girod, and D. Gogny, Comput. Phys. Commun. 63, 365 (1991).

[34] See Supplemental Material at http://link.aps.org/ supplemental/10.1103/PhysRevLett.121.232501 for convergence tests of the $\mathrm{HO}$ basis, for tests of the rigid-rotor approximation to laboratory moments, for a description of the regression analysis and uncertainty estimates, and for the 
full set of detailed and tabulated results, which includes Refs. [35-37].

[35] J. Dobaczewski, W. Nazarewicz, and P.-G. Reinhard, J. Phys. G 41, 074001 (2014).

[36] R. T. Birge, Phys. Rev. 40, 207 (1932).

[37] R. K. Sheline, C. F. Liang, P. Paris, J. Kvasil, and D. Nosek, Phys. Rev. C 51, 1708 (1995).
[38] H. Wollersheim, H. Emling, H. Grein, R. Kulessa, R. Simon, C. Fleischmann, J. de Boer, E. Hauber, C. Lauterbach, C. Schandera, P. Butler, and T. Czosnyka, Nucl. Phys. A556, 261 (1993).

[39] J. Engel, M. Bender, J. Dobaczewski, J. H. de Jesus, and P. Olbratowski, Phys. Rev. C 68, 025501 (2003). 